\title{
RATIONAL MODULES AND HIGHER ORDER CAUCHY TRANSFORMS
}

\author{
JAMES LI-MING WANG \\ Department of Mathematics \\ The University of Alabama \\ University, Alabama 35486 \\ (Received March 24, 1981)
}

ABSTRACT. We apply the higher order Cauchy transforms to describe the closures of rational modules with respect to the $\mathrm{L}^{\mathrm{P}}$ norms, the uniform norm and different Lipschitz norms on a compact set in the plane. KEY WORDS ANV PHRASES. Rational Module, Cauchy Transform 1980 MATHEMATICS SUBJECT CLASSIFICATION CODES. Premary 30A 82, 46399

1. INTRODUCTION.

Let $\mathrm{X}$ be a compact subset of the complex plane. Let the module $R(X) \bar{P}_{m}$ be the space $R+R \bar{z}+\cdots+R_{\bar{z}}^{m}$

$$
=\left\{r_{0}(z)+r_{1}(z) \bar{z}+\cdots \cdot r_{m}(z)^{\frac{m}{z}}\right\},
$$

where each $r_{i}$ is a rational function with poles of $\mathrm{x}$.

In recent years the problem of approximation by rational functions in the areal mean has received great attention (see e.g. [1], [3], [8]) as well as the problem of uniform approximation (see [5], [12]), but little work has been devoted to the corresponding problem of approximation by functions in a rational module due to the lack of analyticity, perhaps. The concept of rational modules arises in a natural fashion when one attempts to study rational approximation in Lipschitz norms. In [10], O'Farrell studied the relation of the problems of approximation by rational modules in different Lipschitz norms, and in the uniform norms, etc., to one another. Not long ago the author proved in [13] that $R(\mathrm{X}) \bar{P}_{1}$ 
is dense in $L^{\mathrm{P}}(\mathrm{X})$ for all $1 \leq \mathrm{p}<\infty$ and $R(\mathrm{X}) \overline{\mathrm{P}}_{2}$ is dense in $\mathrm{C}(\mathrm{X})$ if $\mathrm{X}$ has no interior. For an introductory survey of rational modules and all the standard notations, we refer the readers to the paper of 0 'Farrell [10]. However, the presence of an interior really complicates the situation. Let ${ }_{\mathrm{a}}^{\mathrm{p}}(\mathrm{X})$ be the closed subspace of $\mathrm{L}^{\mathrm{P}}(\mathrm{X})$ which consists of functions analytic in the interior of $\mathrm{X}$. In this note we employ a method of proof which goes back to Brennan [4] to describe the closures of $R(X) \bar{P}_{m}$ in $L^{P}(X)$ and $C(X)$ respectively in terms of the Cauchy transforms of functions in $\mathrm{L}_{a}^{\mathrm{p}}(\mathrm{X})$ for an arbitrary compact subset $x$.

The author is indebted to $\mathrm{J}$. Brennan for valuable conversations and correspondences.

\section{PRELIMINARIES.}

The following notations will be used. If $\mathrm{X}$ is a compact set, $\mathrm{X}$ will be its interior, $\partial X$ its topological boundary. If $V$ is any space of functions on $X$, $[\mathrm{V}]_{\mathrm{p}}$ will be the closure of $\mathrm{V}$ with respect to the $\mathrm{L}^{\mathrm{p}}(\mathrm{X})$ norm and $[\mathrm{V}]_{\mathrm{u}}$ the uniform closure of $\mathrm{V}$ in $\mathrm{C}(\mathrm{X})$.

Let $\mu$ be a (finite Borel) measure on $X$. The Cauchy transform $\hat{\mu}$ is defined by

$$
\hat{\mu}(z)=\int \frac{d \mu(\zeta)}{\zeta-z} .
$$

Some basic properties for $\hat{\mu}$ can be found in [6]. If $g$ is a function on $x$, we will write $\hat{\mathrm{g}}$ for $\widehat{\mathrm{gdm}}$, where $\mathrm{dm}$ denotes the two-dimensional Lebesgue measure.

We write $\mu \perp V$ if $\int \mathrm{fd} \mu=0$ for all $\mathrm{f}$ in $\mathrm{V}$ and $\mathrm{g} \perp \mathrm{V}$ if $\mathrm{gdm} \perp \mathrm{V}$.

The following lemmas play important roles in this theory. Lemma 1 is a special case of the key lemma in [10], and lemma 2 is used by the author in [13].

Throughout this paper, $m$ will be a non-negative integer.

LEMMA 1. Let $\mu$ be a measure on $X$. Then $\mu \perp R(X) \bar{P}_{m+1}$ if and only if $\hat{\mu} \perp R(X) \bar{P}_{\mathrm{m}}$.

LEMMA 2. If $g \in L^{p}(X)$, then $g$ is continuous when $p>2$ and $\hat{\mathrm{g}}$ is continuous when $1<\mathrm{p} \leq 2$.

If $V$ is a space of functions on $X$, we put $\hat{V}=\{\hat{f}: f \varepsilon V\}$. Notice that $f$ is regarded to be zero off X. By induction, we can define the nth Cauchy transform: 
$\hat{\mathrm{V}}_{1}=\hat{\mathrm{V}}, \hat{\mathrm{V}}_{\mathrm{m}}=\left(\hat{\mathrm{V}}_{\mathrm{m}-1}\right)^{\wedge}, \mathrm{m}=2,3,4, \ldots \ldots \ldots \ldots$. In general, we don't have $\mathrm{V} \subset \hat{\mathrm{v}}$, nor $\mathrm{v} \supset \hat{\mathrm{V}}$, on $\mathrm{x}$.

REMARK: As a consequence of Lemma 1, it is not hard to see that

$$
\begin{aligned}
& {\left[R(\mathrm{X}) \overline{\mathrm{P}}_{\mathrm{m}}\right]_{\mathrm{p}}=\left[R(\mathrm{X})+R(\mathrm{X}) \wedge+\cdots+R(\mathrm{X})_{\mathrm{m}}\right]_{\mathrm{p}} \text { and }\left[R(\mathrm{X}) \overline{\mathrm{P}}_{\mathrm{m}}\right]_{\mathrm{u}}=} \\
& {\left[R(\mathrm{X})+R(\mathrm{X}) \hat{\imath}+\cdots+R(\mathrm{X})_{\mathrm{m}}\right]_{\mathrm{u}} \text { for all } \mathrm{m} \geq 1,1 \leq \mathrm{p}<\infty}
\end{aligned}
$$

\section{MAIN RESULTS.}

It is well known that $[R(X)]_{p}={ }_{L}^{p}(x)$ for all $1 \leq \mathrm{p}<2$. This is due to Sinanjan [11] in the case of no interior, to Bers [2] for $p=1$ (and open sets), and to Havin [7] for $1<\mathrm{p}<2$. For $\mathrm{p} \geq 2$, however, $R(\mathrm{X})$ is not always dense in $\mathrm{L}_{\mathrm{a}}^{\mathrm{p}}(\mathrm{X})$. Some necessary and sufficient conditions are given by Brennan [3], Hedberg [8], etc., in terms of various capacities.

In this section, we will show that the higher order Cauchy transforms of $R(\mathrm{X})$ and $\mathrm{L}_{\mathrm{a}}^{\mathrm{p}}(\mathrm{X})$ always have the same closure in $\mathrm{L}^{\mathrm{p}}(\mathrm{X})$ (modulo $R(\mathrm{X})$ ), for all $1 \leq \mathrm{p}<\infty$.

THEOREM 3. Let $\mathrm{X}$ be a compact set. Then $\left[R(\mathrm{X}) \bar{P}_{\mathrm{m}}\right]_{\mathrm{p}}=$ $\left[R(X)+L_{a}^{p}(X)^{\wedge}+\cdots \cdot+L_{a}^{p}(X)_{m}^{\wedge}\right]_{p}$ for all $m \geq 1$ and $1 \leq p<\infty$.

PROOF. It is enough to show that $\left[R(X) \bar{P}_{1}\right]_{p}=\left[R(X)+L_{a}^{p}(X){ }_{p}\right.$ for all $1 \leq \mathrm{p}<\infty$ since the arguments for $\mathrm{m}>1$ are similar.

It is clear that $\left[R(\mathrm{X}) \overline{\mathrm{P}}_{1}\right]_{\mathrm{p}} \subset\left[R(\mathrm{X})+\mathrm{L}_{\mathrm{a}}^{\mathrm{P}}(\mathrm{X}) \wedge_{\mathrm{p}}\right.$ by the remark in section 2 . To prove the other inclusion, we let $\mathrm{g}$ be any function in $\mathrm{L}^{\mathrm{q}}(\mathrm{X}), 1<\mathrm{q} \leq \infty$, $\mathrm{p}^{-1}+\mathrm{q}^{-1}=1$ such that $\mathrm{g} \perp R(\mathrm{X}) \overline{\mathrm{P}}_{1}$. We shall show that $\mathrm{g} \perp \mathrm{L}_{\mathrm{a}}^{\mathrm{p}}(\mathrm{X}) \hat{)}$.

Lemma 1 implies $\hat{g} \perp R(X)$ and therefore $\hat{g}=0$ off $X$. Also $\hat{g}$ is continuous by Lemma 2. It follows that $\hat{\mathrm{g}}=0$ everywhere on $\partial \mathrm{X}$. An argument similar to J. Brennan [4] using the theory of singular integrals and Schwartz lemma concludes that both $\hat{g}$ and $g$ vanishes almost everywhere on $\partial X$. Hence it suffices to prove that $\int_{\frac{Q}{\alpha}} \hat{f g} \mathrm{dm}=0$ for all $\mathrm{f}$ in $\mathrm{L}_{\mathrm{a}}^{\mathrm{p}}(\mathrm{X})$. Again, a similar construction in [4] gives a sequence of functions $\rho_{n}, n=1,2, \cdots$ such that each $\rho_{n}$ has support in $\stackrel{\circ}{X}$ and, writing $\delta$ for $\partial / \partial x+i \partial / \partial y, \bar{\partial} \rho_{n} \rightarrow \hat{g}$ in the $L^{q}(X)$ norm. Thus for any $f$ in $L_{a}^{p}(X)$,

$$
\begin{aligned}
0 & =\lim _{n \rightarrow \infty} \int_{\underset{X}{O}} f\left(\bar{\partial}_{n}\right) d m=\int_{\underset{X}{O}} f \hat{g} d m \\
& =\int_{\underset{X}{g}} \hat{f} g d m .
\end{aligned}
$$


The following theorem shows that, among other things, every nth Cauchy transform of a function in $\mathrm{L}_{\mathrm{a}}^{\mathrm{p}}(\mathrm{X})(\mathrm{n}=2, \ldots, \mathrm{m})$ belongs to the uniform closure of $R(\mathrm{X}) \bar{P}_{\mathrm{m}}$ for all $\mathrm{m} \geq 2,2<\mathrm{p}<\infty$.

THEOREM 4. Let $X$ be a compact set. Then $\left[R(X) \bar{P}_{m}\right]_{u}=[R(X)+$ $\left.R(X)^{\wedge}+L_{a}^{\mathrm{P}}(\mathrm{X})_{2}^{\hat{2}}+\cdots+\mathrm{L}_{\mathrm{a}}^{\mathrm{P}}(\mathrm{X})_{\mathrm{m}}^{\hat{\jmath}}\right]_{\mathrm{u}}$ for all $\mathrm{m} \geq 2$ and $2<\mathrm{p}<\infty$.

PROOF. This follows from Theorem 3 and the fact that $\hat{\mu} \varepsilon L_{\text {loc }}^{q}$ for all $1 \leq \mathrm{q}<2$ and any measure $\mu$ on $\mathrm{x}$ (see [6],p.37)

We may consider the Cauchy transform as a linear map from $c_{c}^{\infty}$, the space of infinitely differentiable functions on the complex plane with compact support, into $C^{\infty}$. In [10], O'Farrell obtains various pairs of norms so that the Cauchy transform is bounded, and thus we have the following corollaries.

COROLLARY 5. Let $\mathrm{X}$ be a compact set. Then $\left[R(X) \bar{P}_{m}\right]_{1 \text { ip } \alpha}=\left[R(X)+R(X)^{\wedge}+L_{a}^{\mathrm{P}}(\mathrm{X})_{2}+\cdots+L_{a}^{\mathrm{p}}(\mathrm{X})_{\mathrm{m}}^{\wedge}\right]_{1 \text { ip } \alpha}$ for all $\mathrm{m} \geq 2,2<\mathrm{p}<\infty$ and $0<\alpha<1$.

COROLLARY 6. Let $X$ be a compact set. Then $\left[R(X) \bar{P}_{m}\right]_{1 \text { ip } 1}=$ $[R(X)+\hat{v}]_{1 \text { ip } 1}$ where $\mathrm{V}$ is any space of functions on $\mathrm{X}$ such that $[\mathrm{V}]_{1 \text { ip } \alpha}=\left[R(\mathrm{X}) \bar{P}_{\mathrm{m}-1}\right\rfloor_{1 \text { ip } \alpha}$, for each fixed $\mathrm{m}$ and $0<\alpha<1$.

REMARK. L. I. Hedberg has pointed out to the author that the problem of approximation in $L^{p}$ by functions in $R(X) \bar{P}_{1}$ is closely related to the problem of approximation by harmonic functions (see [9]).

\section{REFERENCES}

1. T. BAGBY, Quasi topologies and rational approximation, J. Functional Analysis 10 (1972), 259-268.

2. L. BERS, An approximation theorem, J. Analyse Math. 14 (1965), 1-4.

3. J. BRENNAN, Invariant subspaces and rational approximation, J. Functional Analysis 7 (1971), 285-310.

4. J. BRENNAN, Invariant subspaces and weighted polynomial approximation, Ark. mat. 11 (1973), 167-189.

5. T. W. GAMELIN, "Uniform algebras", Prentice Hall, N. J., 1969.

6. J. GARNETT, "Analytic capacity and measure", Springer Lecture Notes, No. $297,1973$.

7. V. P. HAVIN, Approximation in the mean by analytic functions, Soviet Math. Dok1. 9 (1968), 245-248. 
8. L. I. HEDBERG, Approximations in the mean by analytic functions, Trans, Amer. Math. Soc. 163 (1972), 157-171.

9. L. I. HEDBERG, Two approximation problems in function spaces, Ark. Mat. 16 (1978), 51-81.

10. A. O'FARRELL, Annihilators of rational modules. J. Functional Analysis 19(1975) , 373-389.

11. S. 0. SINANJAN, The uniqueness property of analytic functions on closed sets without interior points, Sibirsk. Mat. Z. 6 (1965), 1365-1381.

12. A. G. VITUSHKIN, The analytic capacity of sets in problems of approximation theory, Russian Math. Surveys, 22 (1967), 139-200.

13. J. WANG, Approximation by rational modules on nowhere dense sets, Pac. J. Math., 80 (1979), 293-295. 


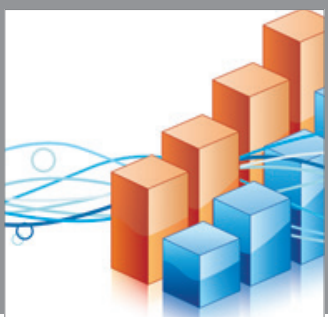

Advances in

Operations Research

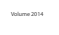

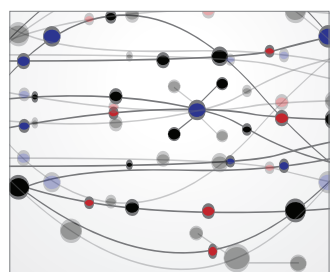

\section{The Scientific} World Journal
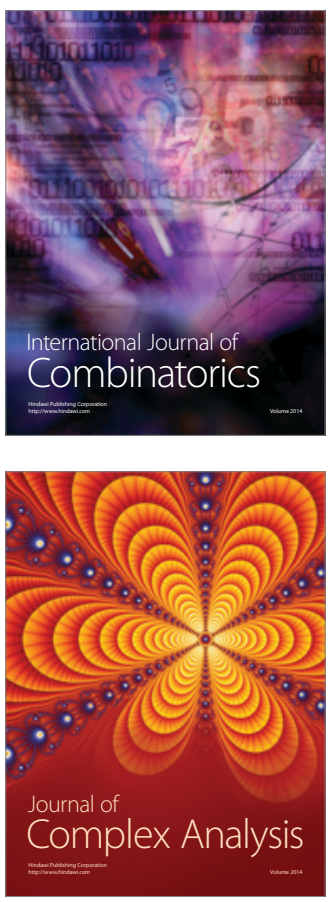

International Journal of

Mathematics and

Mathematical

Sciences
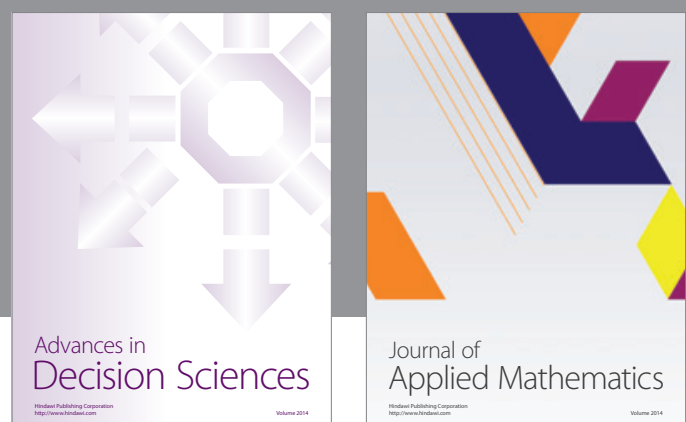

Journal of

Applied Mathematics
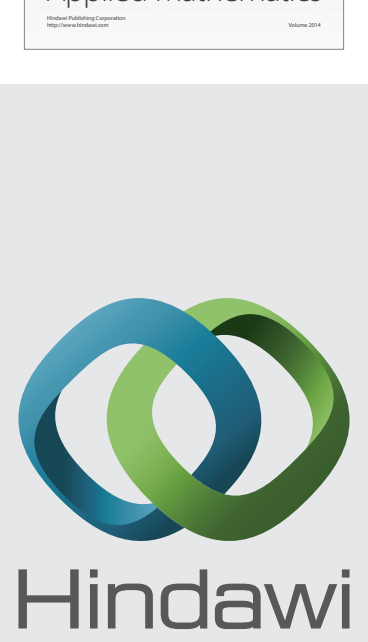

Submit your manuscripts at http://www.hindawi.com
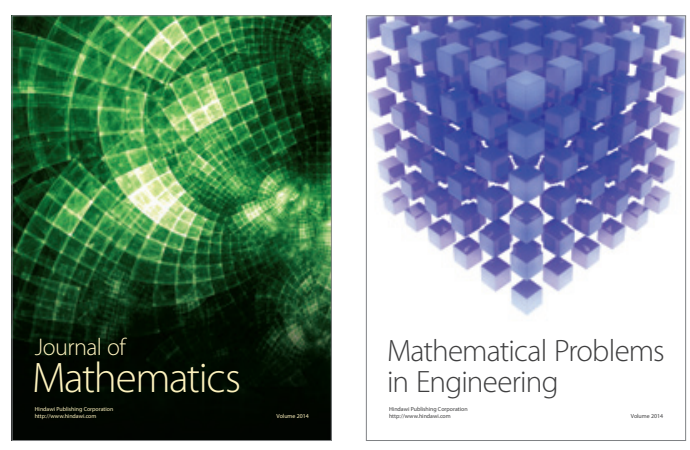

Mathematical Problems in Engineering
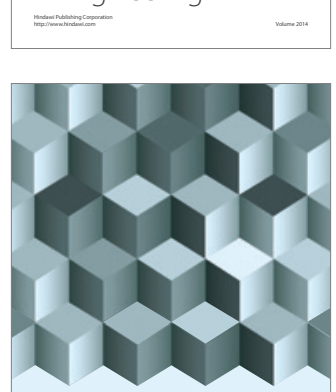

Journal of

Function Spaces
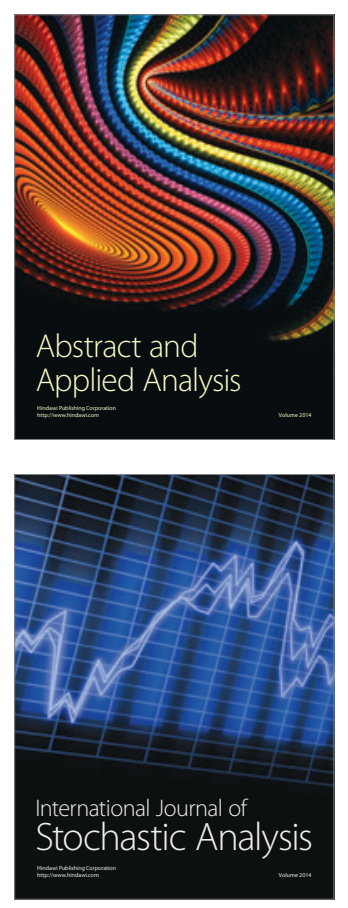

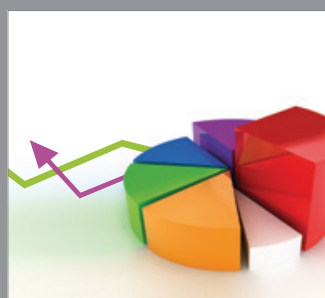

ournal of

Probability and Statistics

Promensencen
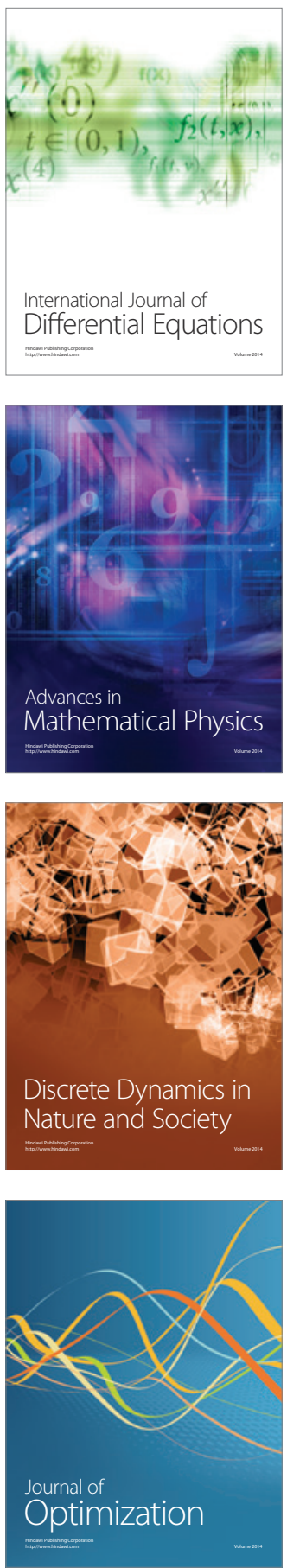